\title{
Modifying the Size of Ultrasound-Induced Liquid- Phase Exfoliated Graphene: from Nanosheets to
}

\section{Nanodots}

Artur Ciesielski,,$^{\dagger}$ Sébastien Haar, ${ }^{\dagger}$ Alessandro Aliprandi, ${ }^{\dagger}$ Mohamed El Garah, ${ }^{\dagger}$ Giulia

Tregnago, ${ }^{\S}$ Giovanni F. Cotella,${ }^{\S}$ Mirella El Gemayel, ${ }^{\dagger}$ Fanny Richard,${ }^{\dagger}$ Haiyan Sun,,${ }^{\ddagger}$ Franco

Cacialli, \$,* Francesco Bonaccorso,

${ }^{\dagger}$ Institut de Science et d’Ingénierie Supramoléculaires (ISIS) and International Center for

Frontier Research in Chemistry (icFRC), Université de Strasbourg and Centre National de la Recherche Scientifique (CNRS), 8 allée Gaspard Monge, 67000 Strasbourg, France. E-mail: samori@unistra.fr

${ }^{\S}$ Department of Physics and Astronomy (CMMP Group) and London Centre for Nanotechnology, University College London, Gower Street London WC1E 6BT, United Kingdom.E-mail: f.cacialli@ucl.ac.uk

${ }^{*}$ Istituto Italiano di Tecnologia, Graphene Labs, Via Morego 30, 16163 Genova, Italy. E-mail: Francesco.Bonaccorso@iit.it

Keywords: graphene, nanodots, liquid-phase exfoliation, photoluminescence. 


\section{ABSTRACT}

Ultrasound induced liquid-phase exfoliation (UILPE) is an established method to produce single(SLG) and few-layer (FLG) graphene nanosheets starting from graphite as precursor. In this paper we investigate the effect of the ultrasonication power in the UILPE process carried out in either $N$-methyl-1,2-pyrrolidone (NMP) or ortho-dichlorobenzene (o-DCB). Our experimental results reveal that while the SLGs/FLGs concentration of the NMP dispersions is independent on the power of the ultrasonic bath during the UILPE process, in $o$-DCB it decreases as the ultrasonication power increases. Moreover, the ultrasonication power has a strong influence on the lateral size of the exfoliated SLGs/FLGs nanosheets in $o$-DCB. In particular, when UILPE is carried out at $\sim 600 \mathrm{~W}$ we obtain dispersions composed of graphene nanosheets with lateral size of $180 \mathrm{~nm}$, whereas at higher power $(\sim 1000 \mathrm{~W})$ we produce graphene nanodots (GNDs) with an average diameter of $\sim 17 \mathrm{~nm}$. The latter nanostructures exhibit a strong and almost excitationindependent photoluminescence emission in the UV/deep-blue region of the electromagnetic spectrum arising from the GNDs intrinsic states and a less intense (and strongly excitation wavelength dependent) emission in the green/red region attributed to defect states. Notably, we also observe visible emission with near-infrared (NIR) excitation at 850 and $900 \mathrm{~nm}$, fingerprint of the presence of up-conversion processes. Overall, our results highlight the crucial importance of the solvents choice for the UILPE process, which under controlled experimental conditions allows the fine-tuning of the morphological properties, such as lateral size and thickness, of the graphene nanosheets towards the realization of luminescent GNDs. 
Graphene and graphene-based composites are at the heart of an ever-growing research effort due to graphene's unique properties, ${ }^{1}$ being extensively explored in a wide range of applications including field-effect transistors (FETs), ${ }^{2-4}$ solar cells, ${ }^{5-7}$ light-emitting diodes (LEDs) ${ }^{8-11}$ touch screens, ${ }^{12}$ electrochemical sensors, ${ }^{13,14}$ batteries,,${ }^{15-18}$ and supercapacitors, $,{ }^{19}, 20$ just to name a few. However, the zero band gap of graphene strongly limits its full exploitation in many photonic and (opto)electronic applications. To open and tune the band gap of graphene, different approaches have been pursued, including covalent functionalization, ${ }^{21-23}$ as well as size confinement either into one-dimensional (1D) nanoribbons ${ }^{24}$ and into zero-dimensional (0D) graphene nanodots (GNDs). ${ }^{25,}{ }^{26}$ Some of the GNDs, and in particular graphene quantum dots (GQDs), produced with the aforementioned approaches, possess well-defined shapes and sizes $(<100 \mathrm{~nm})$, determining quantum-size effect ${ }^{27}$ and excitons confinement. ${ }^{28}$ Typically, GNDs are defined as graphene sheets with lateral dimensions below $100 \mathrm{~nm}$ being single-, two- or a fewlayer thick. ${ }^{26,29}$ Moreover, the fabrication method strongly affect their shape that can vary from circular $^{30}$ to triangular, ${ }^{31,32}$ and hexagonal..$^{32}$ Because of their biocompatibility,,$^{33}$ chemical inertness,${ }^{34}$ low toxicity, ${ }^{35}$ reduced dimensionality, ${ }^{36-38}$ and luminescence, ${ }^{27,39}$ GNDs are emerging as attractive materials for a wide range of applications including electronics and (electrochemi)luminescence sensors..$^{29,40}$

Two different approaches are being pursued for the GNDs production, i.e. synthesis of graphene-like molecules (bottom-up),,$^{30,31,41,42}$ and size reduction processes of graphite ${ }^{33,43}$ or multi-wall carbon fibers ${ }^{44}$ (top-down). On the one hand, bottom-up methods for GNDs production involve stepwise organic synthesis,${ }^{31}$ pyrolysis, ${ }^{45}$ or carbonization of organic precursors. ${ }^{46}$ On the other hand, top-down production of GNDs relies on exposing the starting materials to extremely harsh chemical and/or physical conditions such as those occurring during 


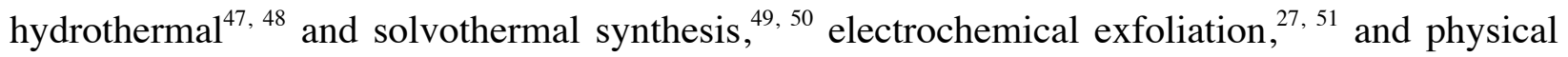
processes such as microwaves irradiation, ${ }^{52}$ and nanolithography ${ }^{53,54}$ Recently, it has been shown that GNDs can be produced via one step chemical reaction following the treatment of carbon fibres in a mixture of $\mathrm{H}_{2} \mathrm{SO}_{4}$ and $\mathrm{HNO}_{3}$ solution. ${ }^{44}$ Moreover, the processes exploiting solvothermal formation of graphite intercalation compounds between graphite powder and sodium potassium tartrate are also being used. ${ }^{55}$ Alternatively, GNDs with a diameter of 4-6 nm were produced by ultrasound induced liquid-phase exfoliation (UILPE) of micronized graphite. ${ }^{33}$ While GNDs can be prepared by exploiting both top-down $n^{31,45,46}$ and bottom-up b. 41, $42^{32}$ approaches, the development of novel and reliable strategies to produce GNDs, having controlled number of functional groups, in large quantities for industrial applications remains challenging. Moreover, although a variety of starting materials has been explored, e.g. micronized graphite, ${ }^{33}$ carbon fibres $^{44}$ or even coal, ${ }^{56}$ the as-produced GNDs still exhibit high level of oxidation of the sheets.

Noteworthy, the UILPE process is one of the most straightforward and up-scalable approach for the production of homogeneous dispersions of non-oxidized graphene sheets, being commonly exploited to extract individual graphene layers from graphite in both organic ${ }^{57-60}$ and aqueous media. ${ }^{61,62}$ Typically, UILPE of graphite is considered as a nondestructive method, with defects caused by the sonication, i.e. fracture of the sheets, mostly located at the edges of the exfoliated graphene sheets, as consequence of their reduced lateral size with respect to the starting material. ${ }^{18,61-63}$ Yet, it has been shown that prolonged ultrasonication time ( $>400$ hours) reduces the sheets lateral size, but also affect the quality of the exfoliated graphene sheets, leading to the presence of a variety of structural defects (e.g. in-plane fracture and/or oxidation) ${ }^{64-66}$ Another critical point of the UILPE is the solvent use for both the exfoliation and 
dispersion process. In fact, ideal solvents for dispersing graphene and graphitic sheets should minimize the interfacial tension between the solvent and the dispersed sheets ${ }^{59}$. Therefore, the choice of the solvent is crucial with, however, very limited selection of solvents that can be used, ${ }^{57,67}$ e.g. $N$-methyl-2-pyrrolidone (NMP) and ortho-dichlorobenzene (o-DCB) amongst the most effective. ${ }^{68}$ This can be ascribed, to a great extent, to the surface tension of NMP and $o$ DCB amounts to $41 \mathrm{mN} \mathrm{m}^{-2}$ and $37 \mathrm{mN} \mathrm{m}^{-2}$, respectively. However, the aforementioned solvents have high boiling points, i.e. $>150^{\circ} \mathrm{C}$, which could be problematic for the deposition onto plastic substrates due to the annealing process required for the solvent removal. ${ }^{69}$

Non-toxic and low-boiling point solvents such as water and alcohols require the addition of stabilizing agents such as polymers or surfactants ${ }^{61,62}$ for the successful exfoliation and dispersion of graphitic sheets. Nevertheless, the presence of such stabilizers in dispersion compromises the (opto)electronic properties of the graphene sheets (such as their photoluminescence -PL- efficiency, i.e. quantum yield), bringing also in this case additional challenges, i.e. their removal, when these dispersions have to be printed onto target substrates. ${ }^{69}$

Importantly, both NMP and $o$-DCB were shown to be able to undergo sonochemical processes such as the formation of free radicals, which are extremely reactive species. ${ }^{70,71}$ In fact, aromatic chlorinated molecules/solvents, such as $o$-DCB, when exposed to high power ultrasound tend to form free radicals. ${ }^{71-74}$ Here, we show that GNDs can be produced through UILPE of bulk graphite powder in $o$-DCB at room temperature. To this end, we explore the influence of the ultrasonication power on the morphology and defectiveness of the exfoliated graphene sheets by tuning the operating power intensities. In particular, we show that with our proposed process we are able to tune the lateral size of the exfoliated graphene nanosheets, e.g. from 200 to $16 \mathrm{~nm}$. We find that produced GNDs (with a diameter of $\sim 17 \mathrm{~nm}$ ) exhibit luminescence properties, with 
a strong and excitation-independent emission in the UV/deep-blue region (i.e. $330-450 \mathrm{~nm}$ ) and a less intense excitation-dependent emission in the green/red region (i.e. $450-650 \mathrm{~nm}$ ). Remarkably, irradiation of these dispersions with near-infrared (NIR) excitations (850 or 900 $\mathrm{nm}$ ) also generates PL peaked at $~ 530 \mathrm{~nm}$, indicative of up-conversion processes.

\section{RESULTS AND DISSCUSION}

To test the impact of the ultrasonication power on the structural quality and lateral size reduction of the exfoliated graphene/graphitic nanosheets, vials containing graphite powder dispersed in NMP and $o$-DCB were treated in bath sonicator for 6 hours at room temperature. The exfoliation experiments were carried out at fixed ultrasound frequency $(40 \mathrm{kHz})$ at two different operating power intensities, i.e. 600 and $1000 \mathrm{~W}$. Subsequently, after the ultrasonication process, the dispersions were allowed to settle for $15 \mathrm{~min} .90 \mathrm{vol} . \%$ and then decanted in the ultracentrifuge tubes and afterward ultracentrifuged, exploiting sedimentationbased separation (SBS) ${ }^{61,69}$ for $30 \mathrm{~min}$ at $5000 \mathrm{rpm}$ followed by another run for $30 \mathrm{~min}$ at 10,000 $\mathrm{rpm}$, see section 4.1 for experimental details. This procedure is carried out to remove thick and un-exfoliated sheets.

$\mathrm{Y}_{\mathrm{W}}(\%)$, which is the yield in weight of the exfoliated graphene/graphitic flakes, defined as the ratio between the weight of dispersed graphitic material and that of the starting graphite flakes ${ }^{1}$ was quantified either through filtration or evaporation of the solvent from the exfoliated material (see Materials and Methods section for details). Figure 1 shows the variation of the $\mathrm{Y}_{\mathrm{W}}$ obtained after the UILPE process. Initially, we have focused our attention on UILPE of graphite powder carried at $600 \mathrm{~W}$. The analysis of 20 independent UILPE experiments revealed that when UILPE is performed at $600 \mathrm{~W}$, comparable values of $\mathrm{Y}_{\mathrm{W}}$ in NMP $(0.8 \%)$, and $o$-DCB $(0.9 \%)$ are 
obtained. Noteworthy, the concentration values of the exfoliated graphitic sheets we obtained cannot be directly compared with those reported in literature, since many experimental parameters such as $\mathrm{Y}_{\mathrm{W}}$, ultrasonication power and temperature are usually not described. Nevertheless, the $\mathrm{Y}_{\mathrm{W}}$ achieved in this work is in good agreement with those previously reported using the same ultrasound bath power $(600 \mathrm{~W})$ and exfoliation time (6 hours) ${ }^{75-77}$

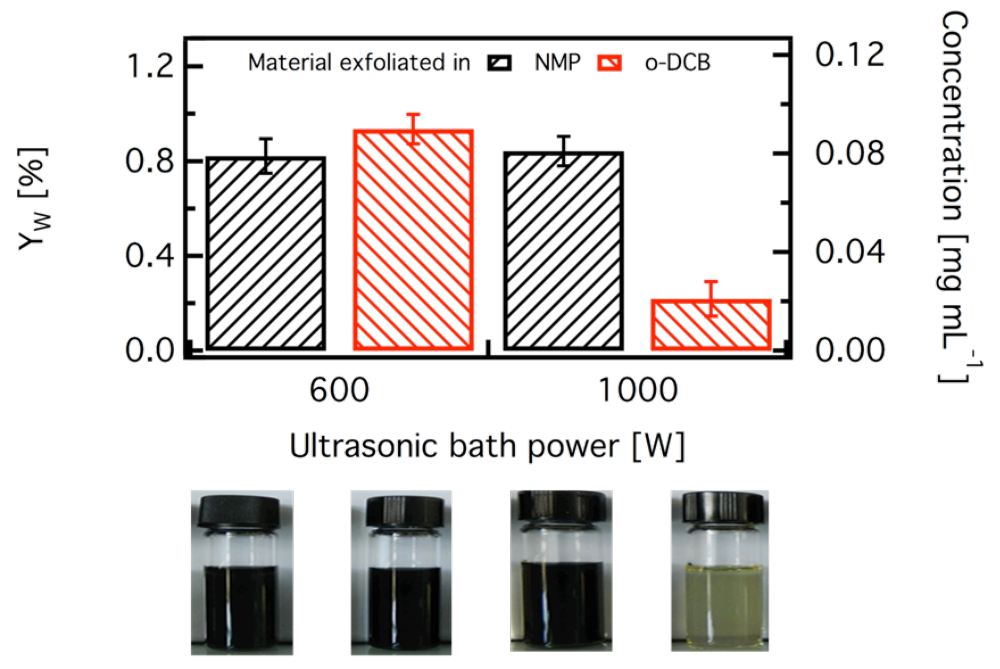

Figure 1. Variation of the $\mathrm{Y}_{\mathrm{W}}$ and concentration of exfoliated materials prepared by UILPE of graphite powder in NMP and $o-\mathrm{DCB}$ at 600 and $1000 \mathrm{~W} . \mathrm{Y}_{\mathrm{W}}$ and the concentration of dispersions were quantified either through filtration or evaporation of the solvent from the exfoliated material - see Methods for details. The error bars reflect a standard deviation of the data as determined by statistical analysis on 20 independent experiments. Representative photographs of dispersions are shown in the bottom panel.

We have than extended our UILPE studies to the experiments carried out at $1000 \mathrm{~W}$ of ultrasonication power. Interestingly, we found that while the $\mathrm{Y}_{\mathrm{W}}$ of NMP-based dispersions does not depend on the power of the ultrasonic bath used during the UILPE, the $\mathrm{Y}_{\mathrm{W}}$ of UILPE in $o$ DCB decreased drastically (see yellow dispersion in Figure 1), achieving a $\mathrm{Y}_{\mathrm{W}}$ as low as $0.19 \%$. 
These results indicate that the power of the ultrasonic bath strongly influences the exfoliation of graphite into SLG/FLG sheets. In order to understand the origin of the reduction in $Y_{w}$ in the case of experiments carried out in $o-\mathrm{DCB}$, we performed other quantitative as well as qualitative analysis of the as obtained dispersions.

High-resolution transmission electron microscopy (HR-TEM) was used to gain qualitative information such as thickness, lateral size and presence of defects in the exfoliated sheets. The analysis of the folded edges is used to estimate the number of layers. ${ }^{78}$ The HR-TEM analysis of the dispersions prepared in $o$-DCB at $600 \mathrm{~W}$ revealed the presence of graphitic sheets (see Figure 2) with average lateral size of $\sim 184 \mathrm{~nm}$ (Figure 2c) and area of $\sim 1.9 \mu \mathrm{m}^{2}$ (Figure 2d). Noteworthy, the examination of the nanosheets' folded edges revealed how the majority of the sheets were 1-2 layers thick (Figure 2e). These results are in good agreement with previous studies on graphene exfoliated at $600 \mathrm{~W} \cdot{ }^{75-77}$
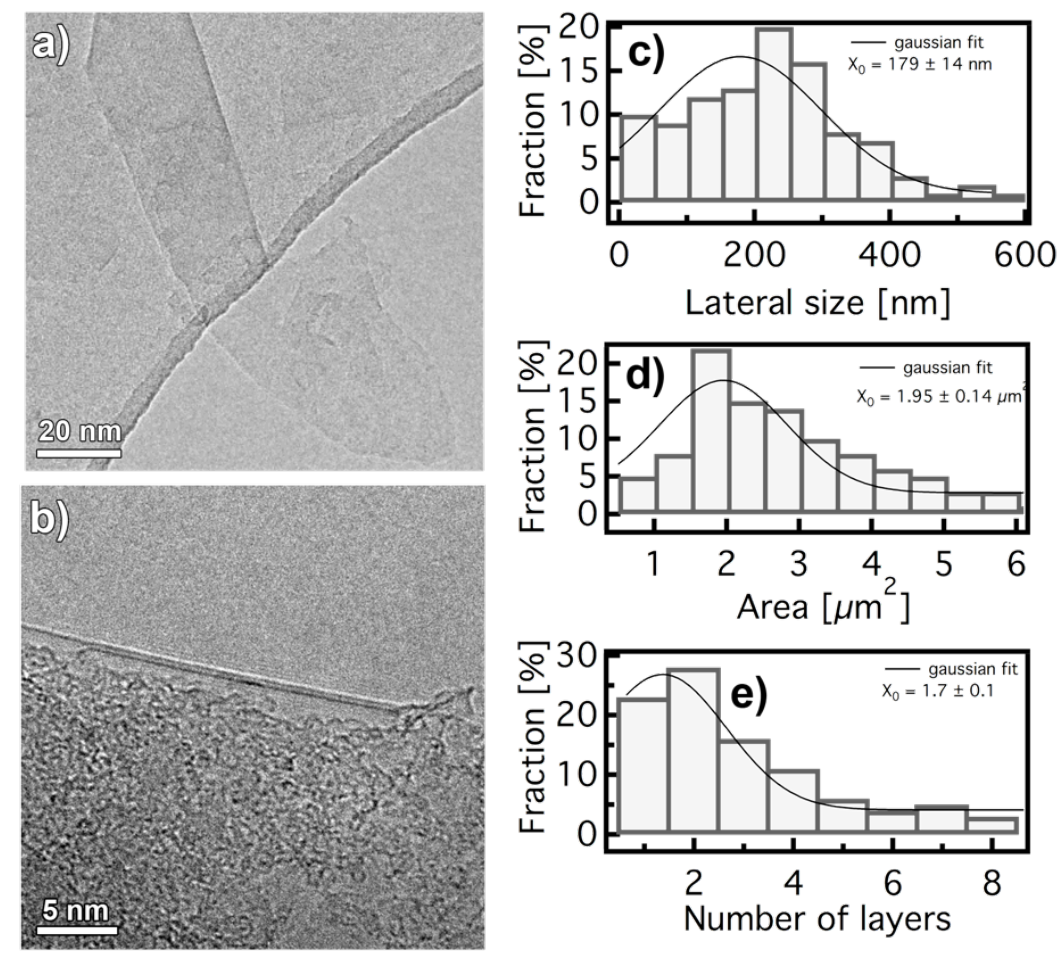
Figure 2. Morphological and statistical analyses of graphene nanosheets prepared through UILPE of graphite powder in $o$-DCB at $600 \mathrm{~W}$. a) TEM micrograph of graphene nanosheet. b) HR-TEM micrograph of the edge of a bi-layer graphene nanosheet. Histograms of the graphene nanosheets: distribution of c) lateral size, d) area, and e) thickness. The fittings have been obtained with a Gaussian distribution.

We have then focused our attention on the dispersions prepared in $o$-DCB at $1000 \mathrm{~W}$. Figure $3 \mathrm{a}$ and $3 \mathrm{~b}$ show the TEM micrographs of GNDs, having average diameter and area of $\sim 17 \mathrm{~nm}$ (see Figure 3e) and $\sim 217 \mathrm{~nm}^{2}$ (Figure 3f), respectively. Here we note that the contrast between GNDs and the carbon coated TEM grids is very low (Figure 3b). Moreover, given the drastically reduced lateral size, the occurrence of regions showing folded GNDs, needed for the thickness estimation, turned out being extremely low. Because of these reasons, the determination of the number of layers by HR-TEM turned out being extremely challenging. To circumvent this problem, the thickness of GNDs could be measured by making use of atomic force microscopy (AFM). Towards this end, GNDs dispersions in $o$-DCB were deposited onto ozone cleaned $\mathrm{SiO}_{2}$ substrates, letting the solvent to evaporate. Both topographical (Figure 3c) and phase images (Figure 3d) show spherical dots, with an average height (Figure 3c) of $0.84 \mathrm{~nm}$, suggesting that the GNDs consist of 1-2 layers. Importantly, while SLG sheets on silicon can show a thickness of $\sim 0.9 \mathrm{~nm}$ (depending on the humidity and the type of the tips), on mica their thickness is lower, resulting in $\sim 0.4 \mathrm{~nm}^{59}$ 

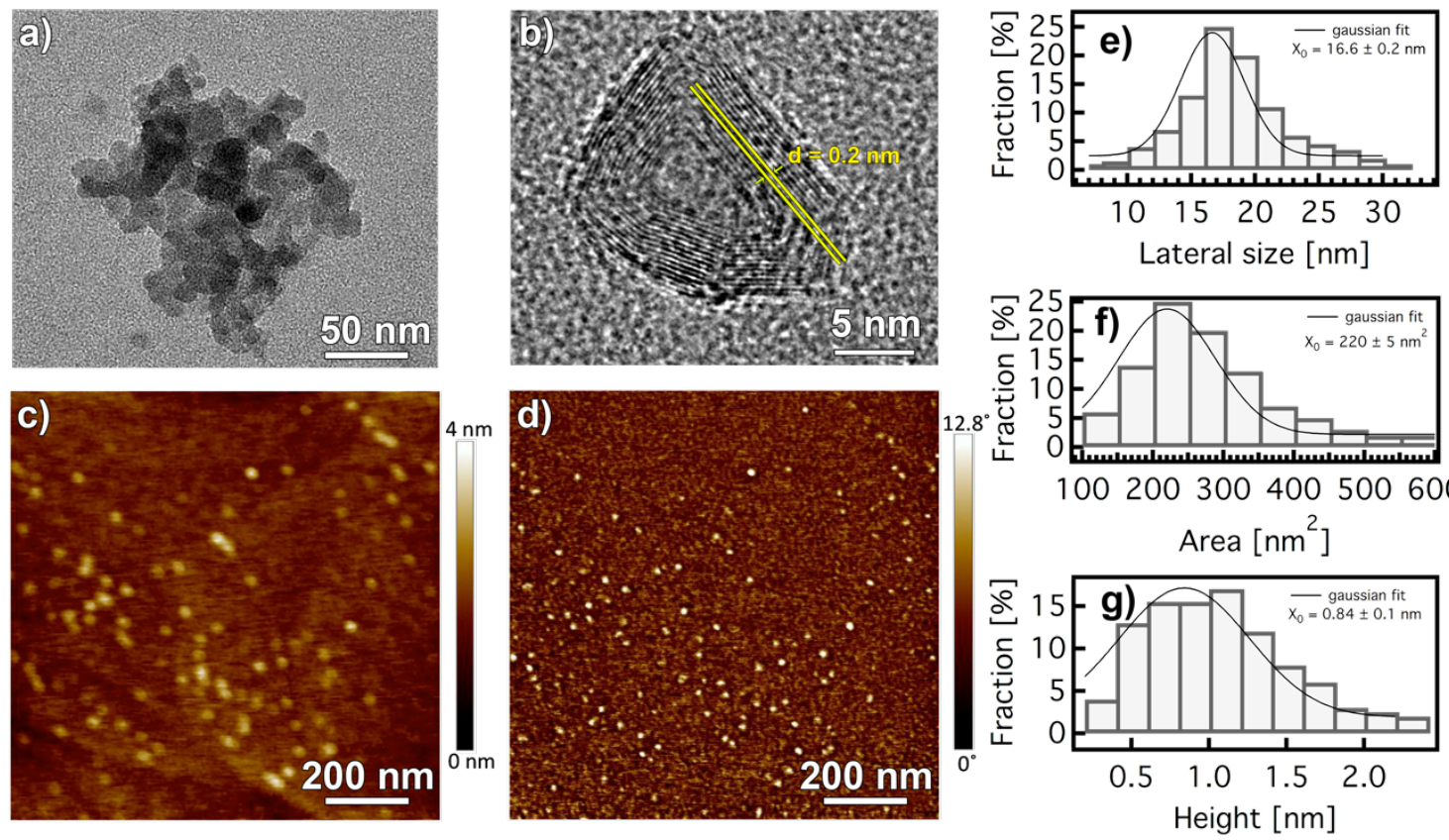

Figure 3. Morphological and corresponding statistical analyses of GNDs prepared by UILPE of graphite powder in $o$-DCB at $1000 \mathrm{~W}$. a) TEM micrograph of aggregated GNDs. b) HR-TEM micrograph of the single GNDs. c) AFM height image of GNDs film, and d) corresponding phase image. Histograms of the GNDs': distribution of e) lateral size, f) area, and g) height. The fittings were obtained with a Gaussian distribution.

These results demonstrate that the power (density) of ultrasounds explored during UILPE of graphite in $o$-DCB significantly affects the lateral size of the exfoliated flakes. This observation can be explained by the fact that aromatic chlorinated solvents, such as $o-\mathrm{DCB}$, when exposed to high power ultrasound tend to form free radicals, ${ }^{71-74}$ which interact with graphene nanosheets by breaking it down into GNDs. In order to exclude possible formation of carbon-based particles through radical coupling between decomposed $o$-DCB, we have performed a series of reference experiments where $o$-DCB have been sonicated in the absence of graphite flakes under the same conditions of experiments previously described. In particular, $10 \mathrm{~mL}$ of o-DCB have been sonicated for 6 hours at room temperature $\left(20^{\circ} \mathrm{C}\right)$, at two different ultrasonication powers, i.e. 
$600 \mathrm{~W}$ and $1000 \mathrm{~W}$. While no visual changes have been monitored for the samples sonicated at $600 \mathrm{~W}$, we have observed a change in color to slight brown of $o$-DCB sonicated at $1000 \mathrm{~W}$, which indicates a possible degradation of the solvent. Our preliminary attempts to characterize the post-sonicated samples failed due to very low yield of the reaction (decomposition). After reproducing the sonication experiments at $1000 \mathrm{~W}$ for multiple times followed by long-lasting evaporation of $o$-DCB we have been able to collect few milligrams of dark powder. We have estimated that the yield by weight of $o$-DCB decomposition under such conditions is lower than $0.01 \%$. Noteworthy, in the case of samples sonicated at $600 \mathrm{~W}$ we have not observed any solid material after solvent evaporation.

We have then performed liquid chromatography-mass spectrometry (LCMS) measurements. LCMS analysis of the dark powder produced at $1000 \mathrm{~W}$ (see Figure S1 of Supplementary Information) points toward the formation of two 1,2-dichloro-4-(3,4-dichlorophenyl)benzene regio-isomers as predominant products $(290 \mathrm{~m} / \mathrm{z}$, see Figure S2). Thus, these results demonstrate that GNDs are not formed by the sono-chemical reaction of o-DCB.

X-ray photoemission spectroscopy (XPS) measurements were carried out to probe the composition of SLG/FLG nanosheets and GNDs, prepared through UILPE in o-DCB at $600 \mathrm{~W}$ and $1000 \mathrm{~W}$, respectively. Figure 4a shows the XPS C1s spectra of SLG/FLG nanosheets, where peaks at $284.7 \mathrm{eV}(\mathrm{C}-\mathrm{C}), 285.8 \mathrm{eV}(\mathrm{C}-\mathrm{O})$ and $288.7 \mathrm{eV}(\mathrm{COOH})$ can be observed. These peaks were also previously reported for UILPE graphene sheets. ${ }^{58}$ Remarkably, the intensity of COOH and $\mathrm{C}-\mathrm{O}$ peaks increases significantly in the case of GNDs (see Figure 4b), which might be associated to the increased circumference/surface area ratio, and in particular to the increased number of edge-based functional groups, such as carbonyl or carboxylic groups, with respect to the graphene sheets. ${ }^{79}$ Clearly, the size reduction of graphitic sheets exfoliated at $1000 \mathrm{~W}$ 
enhances the contribution of the oxygen-containing functional groups at the edges. This is in good agreement with the values observed for other GNDs, ${ }^{44}$ as an intense $\mathrm{COOH}$ peak is also observed at $288.8 \mathrm{eV}$, which results from the edges oxidation during the exfoliation process. Noteworthy, C:O atomic ratios for SLG/FLG nanosheets and GNDs were estimated as 19.2 and 13.7, respectively. In particular the $\mathrm{C}: \mathrm{O}$ ratio of GNDs is remarkably high if compared with the GNDs produced by other top-down approaches, where C:O ratios of 1-10 are typically observed. ${ }^{41-43}$ These results highlight the low oxidative nature of the UILPE process.

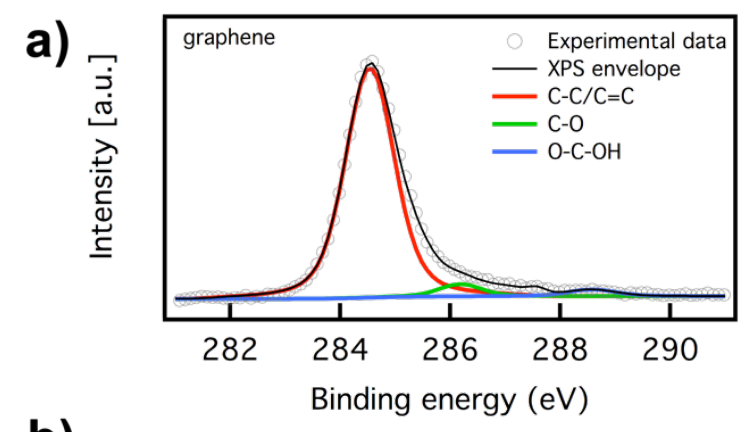

b)
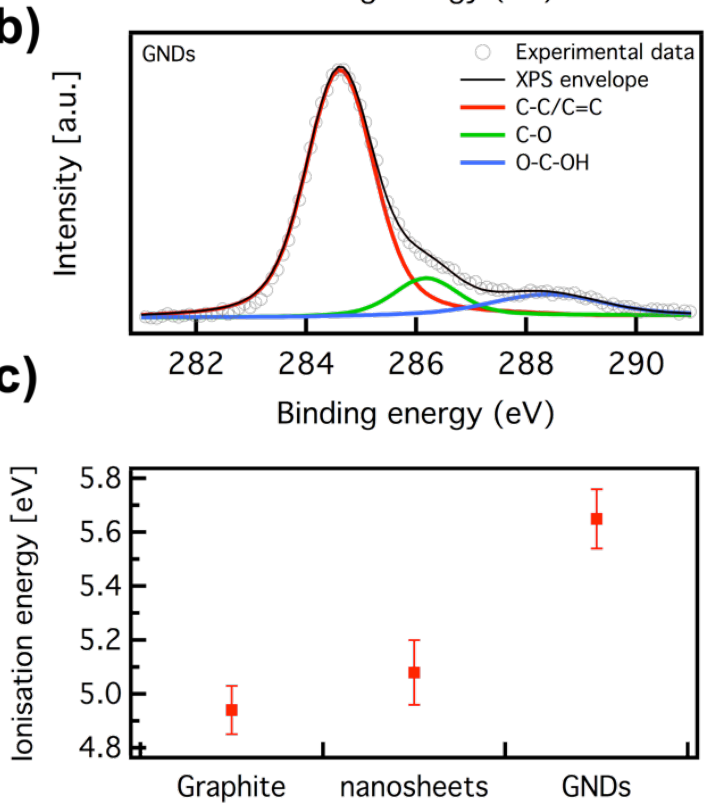

Figure 4. a) C1s XPS spectra of a) SLG/FLG nanosheets, and b) GNDs. c) Variation of ionization energy measured by PhotoElectron Yield counter operated in ambient conditions. 
In order to get further insight onto the energetic levels of the material prepared by UILPE at 600 and $1000 \mathrm{~W}$, we have studied the Ionization Energy (IE) of our samples by means of PhotoElectron Yield counter operated in Ambient conditions (PEYA). We found that the IE is flake size dependent. In fact, IE increases with decreasing the flake size (see Figure 4c), being $5.1 \mathrm{eV}$ and $5.65 \mathrm{eV}$ for graphene nanosheets and GNDs, respectively. The dependence of the IE on the lateral size is in line with theoretical calculation performed on graphene sheets having different nanometric sizes ${ }^{80}$ Particularly, the value measured for GNDs is in the same range as the one calculated for a similar structure, i.e. GQD featuring 42 carbon atoms in the main core. ${ }^{80}$

We then analyzed GNDs by Raman spectroscopy, which is a powerful technique for the investigation of carbon-based materials. ${ }^{78,81-84}$ Figure 5a plots the Raman spectrum measured at $532 \mathrm{~nm}$ excitation wavelength, of representative GNDs deposited on $\mathrm{Si} / \mathrm{SiO}_{2}$ substrate. The $\mathrm{G}$ peak corresponds to the $\mathrm{E}_{2 \mathrm{~g}}$ phonon at the Brillouin zone centre. ${ }^{85}$ The $\mathrm{D}$ peak is due to the breathing modes of $\mathrm{sp}^{2}$ rings, requiring a defect, either structural, i.e. on the basal plane, or at the edges, i.e. by breaking the translational symmetry of the lattice,,${ }^{18,63}$ for its activation by double resonance. ${ }^{78,85,86}$ The $2 \mathrm{D}$ peak is the second order of the $\mathrm{D}$ peak, which is always detected, even in the absence of the D peak. ${ }^{63}$ The 2D is a single peak in SLG, whereas it splits in FLG sheets. This 2D peak splitting reflects the evolution of the electronic band structure of the graphitic sheets. ${ }^{63}$ Double resonance can also happen as intra-valley process, i.e. connecting two points belonging to the same cone around $\mathrm{K}$ or $\mathrm{K}^{,}{ }^{63}$ thus giving origin to the $\mathrm{D}^{\prime}$ peak. Also in this case, as for the $2 \mathrm{D}$ peak, the overtone, i.e. $2 \mathrm{D}^{\prime}$ ' is always detected, even when no $\mathrm{D}^{\prime}$ peak is present. Statistical analysis, based on 20 measurements for each excitation wavelength (532 and $633 \mathrm{~nm}$ ), gave an average position, for the measurements at $532 \mathrm{~nm}$, of the 2D peak, $\operatorname{Pos}(2 \mathrm{D}), \sim 2694 \mathrm{~cm}^{-1}$, while $\operatorname{Pos}(\mathrm{G})$ is peaked at $1585 \mathrm{~cm}^{-1}$. These two peaks, however, are very broad. Indeed, 
FWHM(2D) is $\sim 92 \mathrm{~cm}^{-1}$ while $\operatorname{FWHM(G)}$ is $\sim 45 \mathrm{~cm}^{-1}$, both larger with respect to UILPE graphene sheets, where in average FWHM(2D) is $<70 \mathrm{~cm}^{-1}$ and $\operatorname{FWHM}(\mathrm{G})<25 \mathrm{~cm}^{-1} .{ }^{18,58,63}$ This broadening of the $\mathrm{G}$ and 2D bands can be attributed to both the size- and edge-effects. Indeed, in finite-size domains there is the relaxation of momentum conservation rule ${ }^{63}$ Moreover, as the lateral size of the sheets shrinks, the fractions of edge carbon atoms, which, as aforementioned, act as defect due to the breaking of the translational symmetry of the lattice, increase. Furthermore, as reported by XPS analysis, the GNDs have oxygen-containing functional groups such as $\mathrm{C}=\mathrm{O}$ and $\mathrm{COOH}$. The higher electronegativity of oxygen with respect to carbon results in hole doping of the GNDs, resulting in stiffening of $\operatorname{Pos}(\mathrm{G})$, as well as charge inhomogeneity between edge and basal plane. ${ }^{87}$ The latter could be responsible for the broad G band (FWHM(G) $\left.=45 \mathrm{~cm}^{-1}\right)$ of our GQDs. The as-produced GQDs show also high $\mathrm{I}(\mathrm{D}) / \mathrm{I}(\mathrm{G})$ ratio, 1.20 in average, see Figure 5b). However, this is attributed to the edges of the as-prepared GNDs rather than to the presence of a large amount of structural defects within the basal plane of the GNDs themselves. This observation is supported by the lack of a clear correlation between $I(D) / I(G)$ and $\operatorname{FWHM}(\mathrm{G})$ (see Figure 5b), an indication that the major contribution to the D peak comes from the sample edges. In fact, combining $\mathrm{I}(\mathrm{D}) / \mathrm{I}(\mathrm{G})$ with $\mathrm{FWHM}(\mathrm{G})$ allows us to discriminate between disorder localized at the edges and disorder in the bulk. In the latter case, a higher $\mathrm{I}(\mathrm{D}) / \mathrm{I}(\mathrm{G})$ would correspond to higher FWHM(G). These results, coupled with the XPS data and the morphological characterization, i.e. HRTEM, testify the high quality of the as-produced GNDs. 

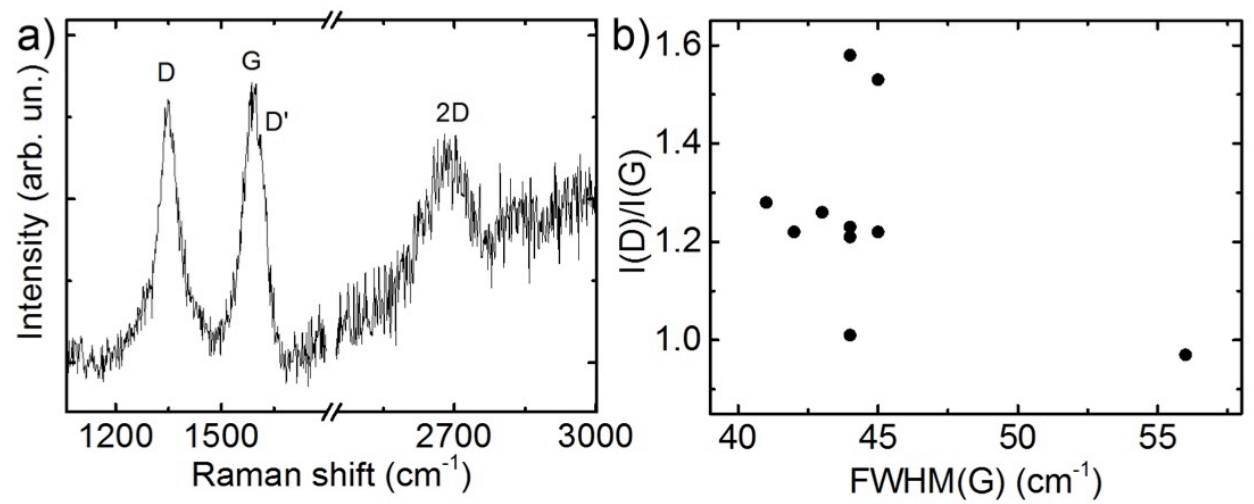

Figure 5. a) Raman spectrum at $532 \mathrm{~nm}$ for representative GNDs. b) Distribution of $\mathrm{I}(\mathrm{D}) / \mathrm{I}(\mathrm{G})$ as a function of $\mathrm{FWHM}(\mathrm{G})$.

To probe the optical properties of GNDs, both PL and photoluminescence excitation (PLE) spectroscopy have been carried out. Figure 6 shows the comparison of PLE and PL emission spectra of GNDs in $o$-DCB at different concentrations in the range of $0.5-5 \mu \mathrm{gL}^{-1}$. Also, given that the decomposition of solvents such as $o$-DCB upon sonication has been demonstrated in the literature, ${ }^{71}$ we report the PL excitation and PL emission spectra of the pure $o$-DCB sonicated at $1000 \mathrm{~W}$ in Figure S4 and the decay of the PL emission in Figure S5 and Table S1, comparing them with those of GNDs to assess any possible influence of the degraded solvent on the optical properties of GNDs. For diluted dispersions $\left(0.5 \mu \mathrm{g} \mathrm{mL^{-1 }}\right.$ see Figure 6 , upper panel) we noted a PL emission in the UV-deep blue region peaked at 340, 370 and $410 \mathrm{~nm}$ when exciting at wavelengths shorter than $350 \mathrm{~nm}$. The PLE observed at $380 \mathrm{~nm}$ shows peaks at 300, 330 and $340 \mathrm{~nm}$. The PLE spectrum is significantly more structured than the absorption spectrum (also reported in Figure 6, upper panel) for the $0.5 \mu \mathrm{g} \mathrm{mL} \mathrm{m}^{-1}$ dispersion (the spectra for the higher concentrations, i.e. 2.5 and $5.0 \mu \mathrm{g} \mathrm{mL}^{-1}$, are also reported for comparison even though saturation occurs in the UV region). The more structured nature of the PLE is interesting, although expected owing to the PL occurring usually from a subset of chromophores, ${ }^{48,88}$ 
compared to absorption. When the PLE is recorded at wavelengths longer than $380 \mathrm{~nm}$, the spectrum becomes broader and featureless. Furthermore, excitation at wavelengths longer than $350 \mathrm{~nm}$ resulted in a broad PL emission at $\sim 500 \mathrm{~nm}$. Such emission red-shifted by $\sim 90 \mathrm{~nm}$ when changing the excitation wavelength from 500 to $400 \mathrm{~nm}$. The excitation wavelength dependence of the emission wavelength and intensity is a well-known phenomenon in GQDs and attributed to excitation of GQDs with different size and/or different emissive sites/domains ${ }^{34,89}$ However, we note that at a concentration of GNDs as low as the one studied here, the emission in the blue/green/red region (i.e. $450-650 \mathrm{~nm}$ ) is weak and species formed upon sonication of $o$-DCB, whose residues may be still present in GNDs, could give a comparable contribution to the emission spectrum (see Figure S4) whereas for higher concentrations the contribution of GNDs becomes dominant (vide infra). The UV/deep-blue (330 - $450 \mathrm{~nm})$ PL emission in GQDs has been previously assigned in the literature to emission from intrinsic states (e.g. caused by the quantum size effect) whereas the green/red peaks (i.e. $450-650 \mathrm{~nm}$ ) has been attributed the emission from GNDs defect states (i.e. presence of oxygen-containing functional groups) ${ }^{55,90}$ Additionally, the absorption spectrum (Figure 6, upper panel) also shows a long tail at longer wavelengths $(>340 \mathrm{~nm})$ that has been attributed to defect states. ${ }^{33}$ We measured a PL quantum efficiency (PLQE) of $(4.7 \pm 0.5 \%)$, in agreement with values reported in literature for GNDs. ${ }^{34}$

The PLE measurements on the 2.5 and $5 \mu \mathrm{g} \mathrm{mL} \mathrm{mL}^{-1}$ dispersions (Figure 6 middle and bottom panels) allow us to follow the evolution of the different peaks as a function of the concentration of the GNDs. In this regard we note that the intensity of the PLE features at wavelengths shorter than $350 \mathrm{~nm}$ decreases strongly with the increase in GNDs concentration. This could be linked either to the formation of aggregate-like species or to inner-filter effects, or to a combination of both of them. Inner filter effects cannot be excluded given that their contribution would be 
important already at low absorbance values. Indeed, the most diluted GND dispersion (0.5 $\mu \mathrm{g}$ $\mathrm{mL}^{-1}$ ) has an optical density of $\sim 0.4$ at $380 \mathrm{~nm}$ and the absorption spectrum has a long tail that extends to wavelengths at which GNDs emit light hence re-absorption effects should be considered. ${ }^{91}$ However, passing from a concentration of 0.5 to $2.5 \mu \mathrm{g} \mathrm{mL}{ }^{-1}$, we also observe a change in the relative intensity of the PLE peaks recorded at an emission wavelength of $380 \mathrm{~nm}$. The intensity of the peak at $300 \mathrm{~nm}$ is lower than those at 330 and $340 \mathrm{~nm}$, contrary to what reported for the more diluted dispersion, i.e. $0.5 \mu \mathrm{g} \mathrm{mL}{ }^{-1}$. Such trend is also reflected in the PL spectrum. The PL intensity in the UV/deep-blue region decreases as the GNDs concentration increases, and alongside we also observe a redistribution of intensity from the component at higher energy (i.e. at 340 and $370 \mathrm{~nm}$ ) to that at lower energies (i.e. at $410 \mathrm{~nm}$ ) when the concentration of GNDs was increased to $2.5 \mu \mathrm{g} \mathrm{mL} \mathrm{m}^{-1}$ At $5 \mu \mathrm{g} \mathrm{mL} \mathrm{m}^{-1}$ (Figure 6 , bottom panel) we observed that the intrinsic state emission in the UV-deep blue region is nearly completely suppressed, as expected for a dispersion with a high concentration of GNDs. Such spectral changes, and in particular the redistribution of intensities between peaks in the PLE, are distinctive features of interchain states ${ }^{92}$ and could not be explained by invoking self-absorption alone. Interchain states, either ground-state or excited-state complexes, could form between GND flakes, or GND flakes and species formed upon sonication of the $o$-DCB (also referred to as sono-polymers), or between $o$-DCB sono-polymers. ${ }^{93}$ Excited-state complexes, either homoor hetero-dimeric, can be excluded because fluorescence decay time is expected to increase by a factor of $\sim 100$ with respect to that of the "isolated" molecule, ${ }^{94}$ therefore one would expect much longer lifetimes rather than the few nanoseconds detected from our samples (Figure S5 and Table S1). Hence, the formation of (weakly-interacting) ground-state complexes, or aggregates, either between GND flake (also supported by TEM images in Figure 3) or the sono-polymer species is 
more likely to occur. We note, however, that the long-lived component in the GNDs dispersion is shorter than that in pure $o$-DCB sonicated at $1000 \mathrm{~W}, \sim 2-2.6 \mathrm{~ns}$ and $3.8 \mathrm{~ns}$ respectively (Table 1) suggesting that aggregates between GNDs are predominant. Furthermore, we note that, in addition to the interaction between two GNDs, subsets of chromophores on a GND flake (possibly split up by a defect due to oxidation) could also interact upon excitation leading to a redistribution of the excitation energy.

We observe a different behavior for the PLE recorded at wavelengths longer than $450 \mathrm{~nm}$ as a

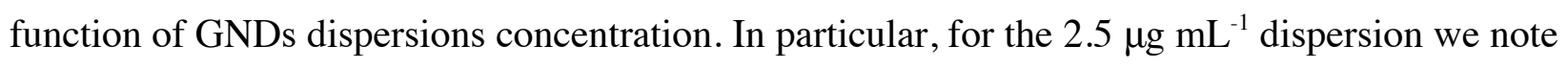
the emergence of a peak at wavelengths between 400 and $500 \mathrm{~nm}$, which is even more evident at higher concentrations $\left(5 \mu \mathrm{g} \mathrm{mL}^{-1}\right)$. Such feature, that is barely visible in the most diluted solution $\left(0.5 \mu \mathrm{g} \mathrm{mL} \mathrm{m}^{-1}\right)$, is totally absent in the $o$-DCB spectrum (Figure S4) and therefore can be attributed, as mentioned above, to the presence of defect states whose emission becomes predominant at high concentration of GNDs. 


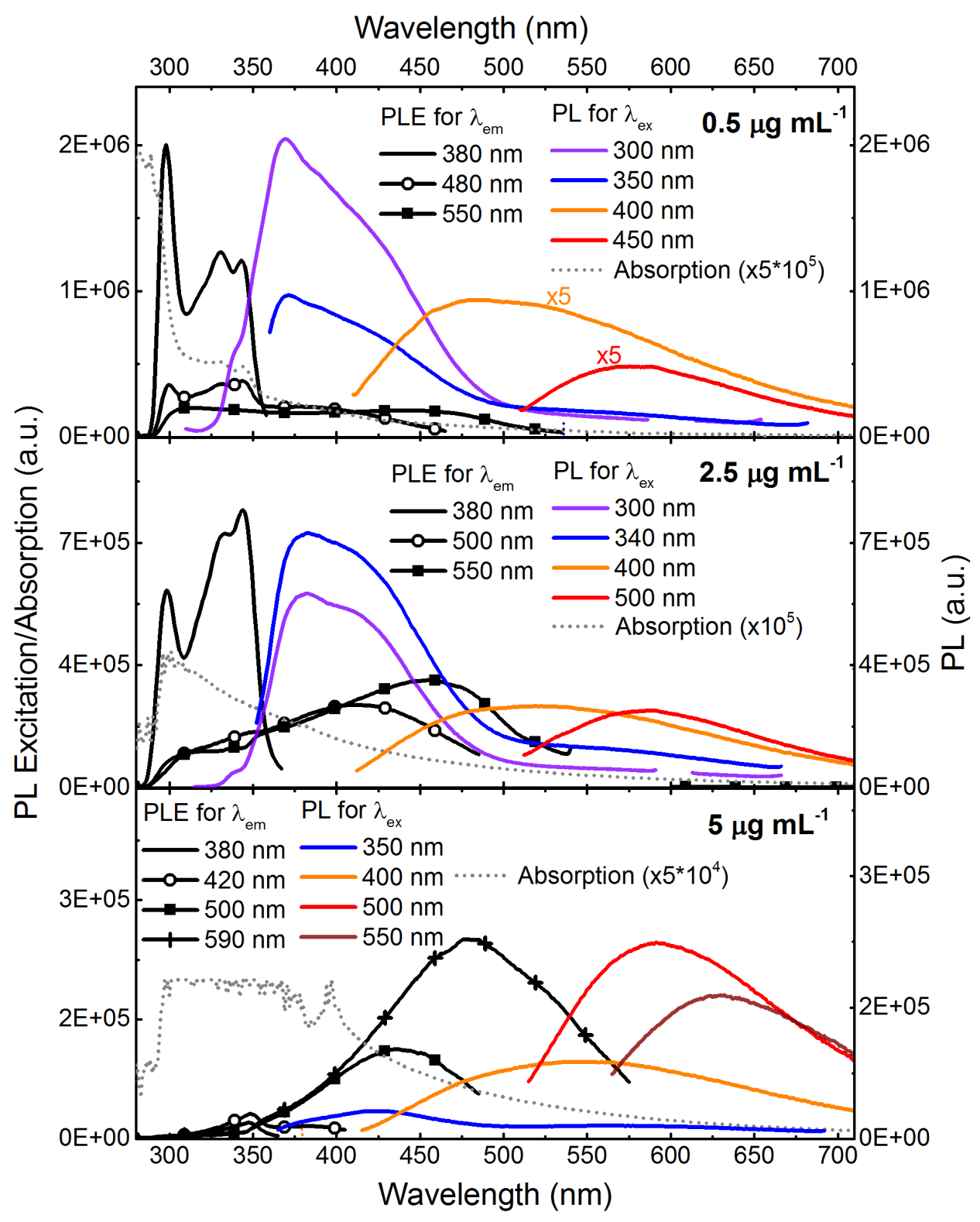

Figure 6. PL excitation and PL emission taken at different emission and excitation for GNDs in $o$-DCB at different concentrations (i.e., $0.5,2.5$ and $5 \mu \mathrm{g} \mathrm{mL} \mathrm{m}^{-1}$ ). The absorption measured in a 1$\mathrm{cm}$ path length cuvette is reported although please note that the spectrum of the 2.5 and $5 \mu \mathrm{g} \mathrm{mL}$ ${ }^{1}$ saturates in the UV region. 


\section{CONCLUSIONS}

In summary, we demonstrated a novel UILPE-based process for the production of GNDs. The proposed approach is cost-effective, with scaling up prospective, as it allows the direct fabrication of GNDs starting from natural graphite by exploiting NMP and $o$-DCB as liquid media. We investigated the UILPE of graphite at $600 \mathrm{~W}$ and $1000 \mathrm{~W}$. We demonstrated that the yield in weight $\left(\mathrm{Y}_{\mathrm{w}}\right)$ of UILPE carried out in NMP does not depend on the ultrasonication power, while it markedly does in $o$-DCB. We reported a substantial decrease of both the $\mathrm{Y}_{\mathrm{W}}$ and the lateral size of the dispersed graphene-based nanomaterials with the increase of the ultrasonication power. In fact, in the case of UILPE carried out at $600 \mathrm{~W}$, we obtained dispersions composed of non-oxidized single- and few-layer graphene nanosheets with a $\mathrm{Y}_{\mathrm{W}}$ of $0.8 \%$. Remarkably, an increase of the power of the ultrasonic bath to $1000 \mathrm{~W}$ resulted in the formation of GNDs at the $\mathrm{Y}_{\mathrm{w}}$ of about $0.19 \%$. These results highlight the importance of the choice of the UILPE solvents that which under certain experimental conditions can undergo sonochemical processes, such as the formation of free radicals, which being highly reactive can fragment the graphene nanosheets into GNDs. In particular, when UILPE was carried out at 600 W dispersions composed of non-oxidized single- and few-layer graphene nanosheets with lateral size of $180 \mathrm{~nm}$ are obtained, whereas when higher power (1000 W) was applied to o-DCB dispersions, GNDs with an average diameter of $\sim 17 \mathrm{~nm}$ were produced. We found that the C:O ratio of these GNDs amounts to 13.7: this is remarkably high if compared to the GNDs produced by other top-down approaches, where the $\mathrm{C}: \mathrm{O}$ ratios of 1-10 are typically observed. Such result highlights the non-oxidative nature of the proposed UILPE process.

The as-produced GNDs dispersed in $o-\mathrm{DCB}$, at a concentration of $0.5 \mu \mathrm{g} \mathrm{mL} \mathrm{m}^{-1}$, have shown a strong and vibronically-structured PL emission in the UV/deep-blue region and a less intense and 
featureless emission at higher wavelengths, which are attributed to intrinsic and defect (and/or aggregate) states, respectively. In addition, GNDs have shown up-conversion PL at $\sim 530 \mathrm{~nm}$ when excited in the near-infrared region. Overall, the as-produced GNDs represent interesting building blocks that thanks to the remarkable optical properties can boost both fundamental studies and applications in photonics and (opto)electronics.

\section{MATERIALS AND METHODS}

Materials. Graphite synthetic flakes (product 332461; batch number 08722AH; + 100 Mesh, $>75 \%$ ), $o$-DCB (product number 240664), and NMP (product number 332461) have been purchased from Sigma Aldrich and used as received.

Synthesis of GNDs. Graphene dispersions were prepared by adding $1 \mathrm{wt} \%$ of graphite powder in $10 \mathrm{~mL}$ of $o$-DCB and NMP followed by 6 hours of ultrasonication at room temperature $\left(20^{\circ} \mathrm{C}\right)$, at two different ultrasonication powers, i.e. $600 \mathrm{~W}$ and $1000 \mathrm{~W}$ by using UltrasonicPower Corporation $10 \mathrm{~L}$ sonic bath operating at $40 \mathrm{kHz}$, equipped with cooling system as well as power intensity and frequency modulation unit. A Hygea Ultrasonic Meter from Ultrawave was used to monitor the power and its constancy throughout the entire process. The samples have been placed in the center of the bath, i.e. in between the two hotspots. Unexfoliated graphitic material has been removed by SBS under centrifugation (Eppendorf 5804, rotor F-34-6-38, $30 \mathrm{~min}$ at $5000 \mathrm{rpm}$ followed by $30 \mathrm{~min}$ at 10,000 rpm), yielding homogeneous dispersions.

$Y_{w}$ and the concentration of exfoliated material. To quantify the $Y_{w}$ and the concentration of SLGs/FLGs and GNDs after centrifugation two different protocols have been followed: i) dispersion prepared at $600 \mathrm{~W}$ - a mixture of exfoliated material and chloroform $\left(\mathrm{CHCl}_{3}\right)$ was first 
warmed up to $50{ }^{\circ} \mathrm{C}$ for $30 \mathrm{~min}$ and then passed through polytetrafluoroethylene (PTFE) membrane filters (pore size $100 \mathrm{~nm}$ ). The remaining solvent molecules were washed several times with diethyl ether and $\mathrm{CHCl}_{3}$; ii) dispersion prepared at $1000 \mathrm{~W}$ - in the case of the dispersions prepared in $o$-DCB at $1000 \mathrm{~W}$, all the exfoliated graphitic material was filtered through the $100 \mathrm{~nm}$ pore size PTFE membranes. Afterward, the dispersions were placed in an oven $\left(100{ }^{\circ} \mathrm{C}\right)$ under vacuum for 3 days for the solvent evaporation. Measurements of the filtered and dried materials were performed on a microbalance (Sartorius MSA2.75) to quantify the concentration of the exfoliated graphitic material.

Transmission Electron Microscopy. $5 \mu 1$ of dispersion containing exfoliated graphitic material was deposited on a $10 \mathrm{~nm}$ thick carbon film coated on a $400 \mathrm{Cu}$ mesh grid. After $1 \mathrm{~min}$ of adsorption, another $5 \mu 1$ drop of dispersion was deposited. After reproducing 3 times the same procedure, TEM grids were dried under vacuum at room temperature. The TEM images were recorded on a Philips CM120 Transmission Electron Microscope operating at $100 \mathrm{kV}$ with a LaB6 cathode. Areas of structures of interest were recorded at a magnification of $120 \times$ on a Peltier cooled scan CCD camera. High Resolution TEM images were recorded on a FEI Tecnai 20 TEM operating at $200 \mathrm{kV}$ at a magnification of $300 \times$ on a Gatan Ultrascan $1000 \mathrm{CCD}$ camera $2048 \times 2048$ px.

Photoluminescence analysis. The optical properties were further investigated for GNDs produced in $o$-DCB at a ultrasonication power of $1000 \mathrm{~W}$. As-prepared GNDs were re-dispersed

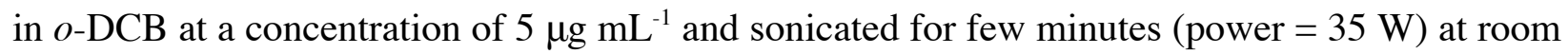
temperature. The dispersions were filtered using a $0.20 \mu \mathrm{m}$ filter and diluted 2 and 10 times. The steady-state PL and PLE spectra were recorded with FluoroMax-4 (Horiba Jobin Yvon) spectrofluorometer with detection in the $200-850 \mathrm{~nm}$ range. The PLQE was measured relative 
to a standard reference material with a known PLQE (quinine sulphate de-hydrate in $1.0 \mathrm{~N}$ sulphuric acid, PLQE of (54.6 \pm 5$) \%$, NIST standard reference material SRM\# 936a, www.nist.gov/srm).

Raman spectroscopy. Raman measurements were carried out with a Renishaw 1000 at 532 $\mathrm{nm}$ and $632.8 \mathrm{~nm}$ and a $100 \mathrm{X}$ objective, with an incident power of $\approx 1 \mathrm{~mW}$. The $\mathrm{D}, \mathrm{G}$, and $2 \mathrm{D}$ peaks were fitted with Lorentzian functions.

Atomic Force Microscopy. AFM morphological characterization was performed in tapping mode in a Dimension 3100 microscope equipped with a Nanoscope IV controller (Digital Instruments). Commercial silicon cantilevers with a nominal spring constant of $40 \mathrm{~N} / \mathrm{m}$ were used for morphological characterization in tapping mode.

X-ray photoelectron spectroscopy. XPS analyses were carried out on a Thermo Scientific KAlpha X-ray photoelectron spectrometer with a basic chamber pressure of $\sim 10^{-9}$ mbar and an $\mathrm{Al}$ anode as the X-ray source (x-ray radiation of $1486 \mathrm{eV}$ ). Spot sizes of $400 \mu \mathrm{m}$ were used and pass energies of $200.00 \mathrm{eV}$ for survey scans and $50.00 \mathrm{eV}$ for high-resolution scans were used. 150 $\mu \mathrm{L}$ of dispersions were spin coated on Au substrate for 1 minute at $1000 \mathrm{rpm}$ and substrates were annealed for 1 day at $100^{\circ} \mathrm{C}$ in an oven under vacuum.

Photo-Electron Yield counter operating in Ambient conditions (PEYA). Ambient photoelectron spectroscopy measurements were performed by sampling in each measurement an area of about $4 \mathrm{~mm}^{2}$ (beam size) by using a PhotoElectron Yield counter operating in Ambient conditions (PEYA), Model AC-2 from Riken Keike Co.

\section{AUTHOR INFORMATION}

Conflict of Interest: The authors declare no competing financial interest. 


\section{Corresponding Author}

*Address correspondence to samori@ unistra.fr

\section{ACKNOWLEDGMENT}

This work was supported by the European Commission through the Graphene Flagship - Core

1 project (GA-696656) and the Marie-Curie ITN projects GENIUS (GA-264694), SYNCHRONICS (GA-643238) and iSwitch (GA-642196). We also thank the support of the Agence Nationale de la Recherche through the LabEx CSC (ANR-10-LABX-0026_CSC), the International Center for Frontier Research in Chemistry (icFRC), as well as the Royal Society. FC is a Royal Society Wolfson Research Merit Award holder.

Supporting Information Available: Liquid chromatography-mass spectrometry (LCMS) and

${ }^{1} \mathrm{H}-\mathrm{NMR}$ analyses of the sonochemical degradation of $o$-DCB. Optical properties of $o$-DCB sonicated at $1000 \mathrm{~W}$. This material is available free of charge via the Internet at http://pubs.acs.org.

\section{REFERENCES}

1. Ferrari, A. C.; Bonaccorso, F.; Fal'ko, V.; Novoselov, K. S.; Roche, S.; Boggild, P.; Borini, S.; Koppens, F. H.; Palermo, V.; Pugno, N.; Garrido, J. A.; Sordan, R.; Bianco, A.; Ballerini, L.; Prato, M.; Lidorikis, E.; Kivioja, J.; Marinelli, C.; Ryhanen, T.; Morpurgo, A., et $a l$. Science and Technology Roadmap for Graphene, Related Two-Dimensional Crystals, and Hybrid Systems. Nanoscale 2015, 7, 4598-4810.

2. $\quad$ El Gemayel, M.; Haar, S.; Liscio, F.; Schlierf, A.; Melinte, G.; Milita, S.; Ersen, O.; Ciesielski, A.; Palermo, V.; Samorì, P. Leveraging the Ambipolar Transport in Polymeric FieldEffect Transistors via Blending with Liquid-Phase Exfoliated Graphene. Adv. Mater. 2014, 26, 4814-4819.

3. Mosciatti, T.; Haar, S.; Liscio, F.; Ciesielski, A.; Orgiu, E.; Samorì, P. A Multifunctional Polymer-Graphene Thin-Film Transistor with Tunable Transport Regimes. Acs Nano 2015, 9 , 2357-236. 
4. Fiori, G.; Bonaccorso, F.; Iannaccone, G.; Palacios, T.; Neumaier, D.; Seabaugh, A.; Banerjee, S. K.; Colombo, L. Electronics Based on Two-Dimensional Materials. Nat.

Nanotechnol. 2014, 9, 768-779.

5. $\quad$ Wang, J. T. W.; Ball, J. M.; Barea, E. M.; Abate, A.; Alexander-Webber, J. A.; Huang, J.; Saliba, M.; Mora-Sero, I.; Bisquert, J.; Snaith, H. J.; Nicholas, R. J. Low-Temperature Processed Electron Collection Layers of Graphene/TiO2 Nanocomposites in Thin Film Perovskite Solar Cells. Nano Lett. 2014, 14, 724-730.

6. Habisreutinger, S. N.; Leijtens, T.; Eperon, G. E.; Stranks, S. D.; Nicholas, R. J.; Snaith, H. J. Carbon Nanotube/Polymer Composites as a Highly Stable Hole Collection Layer in Perovskite Solar Cells. Nano Lett. 2014, 14, 5561-5568.

7. Chang, H. X.; Wu, H. K. Graphene-Based Nanomaterials: Synthesis, Properties, and Optical and Optoelectronic Applications. Adv. Funct. Mater. 2013, 23, 1984-1997.

8. Withers, F.; Del Pozo-Zamudio, O.; Mishchenko, A.; Rooney, A. P.; Gholinia, A.; Watanabe, K.; Taniguchi, T.; Haigh, S. J.; Geim, A. K.; Tartakovskii, A. I.; Novoselov, K. S. Light-Emitting Diodes by Band-Structure Engineering in van der Waals Heterostructures. Nat. Mater. 2015, 14, 301-306.

9. Johannsen, J. C.; Ulstrup, S.; Crepaldi, A.; Cilento, F.; Zacchigna, M.; Miwa, J. A.; Cacho, C.; Chapman, R. T.; Springate, E.; Fromm, F.; Raidel, C.; Seyller, T.; King, P. D.; Parmigiani, F.; Grioni, M.; Hofmann, P. Tunable Carrier Multiplication and Cooling in Graphene. Nano Lett. 2015, 15, 326-331.

10. Brinkhaus, L.; Katsukis, G.; Malig, J.; Costa, R. D.; Garcia-Iglesias, M.; Vazquez, P.; Torres, T.; Guldi, D. M. Tuning the Stability of Graphene Layers by Phthalocyanine-Based oPPV Oligomers Towards Photo- and Redoxactive Materials. Small 2013, 9, 2348-2357.

11. Bausi, F.; Schlierf, A.; Treossi, E.; Schwab, M. G.; Palermo, V.; Cacialli, F. Thermal Treatment and Chemical Doping of Semi-Transparent Graphene Films. Org. Electron. 2015, 18, 53-60.

12. Bonaccorso, F.; Sun, Z.; Hasan, T.; Ferrari, A. C. Graphene Photonics and Optoelectronics. Nature Photon. 2010, 4, 611-622.

13. Zhang, M.; Liao, C. Z.; Mak, C. H.; You, P.; Mak, C. L.; Yan, F. Highly Sensitive Glucose Sensors Based on Enzyme-Modified Whole-Graphene Solution-Gated Transistors. Sci. Rep. 2015, 5, 8311 .

14. Pumera, M. Graphene-Based Nanomaterials for Energy Storage. Energy Environ. Sci. 2011, 4, 668-674.

15. Bonaccorso, F.; Colombo, L.; Yu, G. H.; Stoller, M.; Tozzini, V.; Ferrari, A. C.; Ruoff, R. S.; Pellegrini, V. Graphene, Related Two-Dimensional Crystals, and Hybrid Systems for Energy Conversion and Storage. Science 2015, 347, 1246501.

16. Yu, D. S.; Goh, K.; Wang, H.; Wei, L.; Jiang, W. C.; Zhang, Q.; Dai, L. M.; Chen, Y. Scalable Synthesis of Hierarchically Structured Carbon Nanotube-Graphene Fibres for Capacitive Energy Storage. Nat. Nanotechnol. 2014, 9, 555-562.

17. Park, K. H.; Lee, D.; Kim, J.; Song, J.; Lee, Y. M.; Kim, H. T.; Park, J. K. Defect-Free, Size-Tunable Graphene for High-Performance Lithium Ion Battery. Nano Lett. 2014, 14, 43064313.

18. Hassoun, J.; Bonaccorso, F.; Agostini, M.; Angelucci, M.; Betti, M. G.; Cingolani, R.; Gemmi, M.; Mariani, C.; Panero, S.; Pellegrini, V.; Scrosati, B. An Advanced Lithium-Ion Battery Based on a Graphene Anode and a Lithium Iron Phosphate Cathode. Nano Lett. 2014, 14, 4901-4906. 
19. Mahmood, N.; Zhang, C. Z.; Yin, H.; Hou, Y. L. Graphene-Based Nanocomposites for Energy Storage and Conversion in Lithium Batteries, Supercapacitors and Fuel Cells. J. Mat . Chem. A 2014, 2, 15-32.

20. Han, S.; Wu, D. Q.; Li, S.; Zhang, F.; Feng, X. L. Porous Graphene Materials for Advanced Electrochemical Energy Storage and Conversion Devices. Adv. Mater. 2014, 26, 849864.

21. Choi, J.; Lee, H.; Kim, K. J.; Kim, B.; Kim, S. Chemical Doping of Epitaxial Graphene by Organic Free Radicals. J. Phys. Chem. Lett. 2010, 1, 505-509.

22. Wang, X. R.; Li, X. L.; Zhang, L.; Yoon, Y.; Weber, P. K.; Wang, H. L.; Guo, J.; Dai, H. J. N-Doping of Graphene Through Electrothermal Reactions with Ammonia. Science 2009, 324, 768-771.

23. Choi, J.; Kim, K. J.; Kim, B.; Lee, H.; Kim, S. Covalent Functionalization of Epitaxial Graphene by Azidotrimethylsilane. J. Phys. Chem. C 2009, 113, 9433-9435.

24. Chen, L.; Hernandez, Y.; Feng, X. L.; Müllen, K. From Nanographene and Graphene Nanoribbons to Graphene Sheets: Chemical Synthesis. Angew. Chem. Int. Ed. 2012, 51, 76407654.

25. Kim, S.; Hwang, S. W.; Kim, M. K.; Shin, D. Y.; Shin, D. H.; Kim, C. O.; Yang, S. B.; Park, J. H.; Hwang, E.; Choi, S. H.; Ko, G.; Sim, S.; Sone, C.; Choi, H. J.; Bae, S.; Hong, B. H. Anomalous Behaviors of Visible Luminescence from Graphene Quantum Dots: Interplay between Size and Shape. Acs Nano 2012, 6, 8203-8208.

26. Roy, P.; Chen, P. C.; Periasamy, A. P.; Chen, Y. N.; Chang, H. T. Photoluminescent Carbon Nanodots: Synthesis, Physicochemical Properties and Analytical Applications. Mater. Today 2015, 18, 447-458.

27. Li, Y.; Hu, Y.; Zhao, Y.; Shi, G. Q.; Deng, L. E.; Hou, Y. B.; Qu, L. T. An Electrochemical Avenue to Green-Luminescent Graphene Quantum Dots as Potential ElectronAcceptors for Photovoltaics. Adv. Mater. 2011, 23, 776-780.

28. Einevoll, G. T. Confinement of Excitons in Quantum Dots. Phys. Rev. B 1992, 45, 34103417.

29. Sun, H. J.; Wu, L.; Wei, W. L.; Qu, X. G. Recent Advances in Graphene Quantum Dots for Sensing. Mater. Today 2013, 16, 433-442.

30. Liu, R. L.; Wu, D. Q.; Feng, X. L.; Müllen, K. Bottom-Up Fabrication of

Photoluminescent Graphene Quantum Dots with Uniform Morphology. J. Am. Chem. Soc. 2011, 133, 15221-15223.

31. Yan, X.; Cui, X.; Li, L. S. Synthesis of Large, Stable Colloidal Graphene Quantum Dots with Tunable Size. J. Am. Chem. Soc. 2010, 132, 5944-5945.

32. Lu, J.; Yeo, P. S. E.; Gan, C. K.; Wu, P.; Loh, K. P. Transforming C-60 Molecules into Graphene Quantum Dots. Nat. Nanotechnol. 2011, 6, 247-252.

33. Liu, F.; Jang, M. H.; Ha, H. D.; Kim, J. H.; Cho, Y. H.; Seo, T. S. Facile Synthetic Method for Pristine Graphene Quantum Dots and Graphene Oxide Quantum Dots: Origin of Blue and Green Luminescence. Adv. Mater. 2013, 25, 3657-3662.

34. Li, L. L.; Wu, G. H.; Yang, G. H.; Peng, J.; Zhao, J. W.; Zhu, J. J. Focusing on Luminescent Graphene Quantum Dots: Current Status and Future Perspectives. Nanoscale 2013, 5, 4015-4039.

35. Zhu, S. J.; Zhang, J. H.; Qiao, C. Y.; Tang, S. J.; Li, Y. F.; Yuan, W. J.; Li, B.; Tian, L.; Liu, F.; Hu, R.; Gao, H. N.; Wei, H. T.; Zhang, H.; Sun, H. C.; Yang, B. Strongly Green- 
Photoluminescent Graphene Quantum Dots for Bioimaging Applications. Chem. Commun. 2011, 47, 6858-6860.

36. Lim, S. Y.; Shen, W.; Gao, Z. Q. Carbon Quantum Dots and Their Applications. Chem. Soc. Rev. 2015, 44, 362-381.

37. Zheng, X. T.; Ananthanarayanan, A.; Luo, K. Q.; Chen, P. Glowing Graphene Quantum

Dots and Carbon Dots: Properties, Syntheses, and Biological Applications. Small 2014, 11, 1620-1636.

38. Bacon, M.; Bradley, S. J.; Nann, T. Graphene Quantum Dots. Part. Part. Sys. Char. 2014, 31, 415-428.

39. Zhuo, S.; Shao, M.; Lee, S. T. Upconversion and Downconversion Fluorescent Graphene Quantum Dots: Ultrasonic Preparation and Photocatalysis. Acs Nano 2012, 6, 1059-1064.

40. Li, P.; Di Stasio, F.; Eda, G.; Fenwick, O.; McDonnell, S. O.; Anderson, H. L.;

Chhowalla, M.; Cacialli, F. Incorporation of Graphene Oxide Quantum Dots in Light-Emitting

Diodes Based on a Water Soluble Emissive Polymer. Chemphyschem 2015, 16, 1258-1262

41. Umrao, S.; Jang, M. H.; Oh, J. H.; Kim, G.; Sahoo, S.; Cho, Y. H.; Srivastva, A.; Oh, I.

K. Microwave Bottom-up Route for Size-Tunable and Switchable Photoluminescent Graphene Quantum Dots Using Acetylacetone: New Platform for Enzyme-Free Detection of Hydrogen Peroxide. Carbon 2015, 81, 514-524.

42. Dong, Y. Q.; Shao, J. W.; Chen, C. Q.; Li, H.; Wang, R. X.; Chi, Y. W.; Lin, X. M.; Chen, G. N. Blue Luminescent Graphene Quantum Dots and Graphene Oxide Prepared by Tuning the Carbonization Degree of Citric Acid. Carbon 2012, 50, 4738-4743.

43. Buzaglo, M.; Shtein, M.; Regev, O. Graphene Quantum Dots Produced by Microfluidization. Chem. Mater. 2016, 28, 21-24.

44. Peng, J.; Gao, W.; Gupta, B. K.; Liu, Z.; Romero-Aburto, R.; Ge, L. H.; Song, L.; Alemany, L. B.; Zhan, X. B.; Gao, G. H.; Vithayathil, S. A.; Kaipparettu, B. A.; Marti, A. A.; Hayashi, T.; Zhu, J. J.; Ajayan, P. M. Graphene Quantum Dots Derived from Carbon Fibers. Nano Lett. 2012, 12, 844-849.

45. Atienzar, P.; Primo, A.; Lavorato, C.; Molinari, R.; Garcia, H. Preparation of Graphene Quantum Dots from Pyrolyzed Alginate. Langmuir 2013, 29, 6141-6146.

46. Lai, S. K.; Luk, C. M.; Tang, L.; Teng, K. S.; Lau, S. P. Photoresponse of PolyanilineFunctionalized Graphene Quantum Dots. Nanoscale 2015, 7, 5338-5343.

47. Jin, S. H.; Kim, D. H.; Jun, G. H.; Hong, S. H.; Jeon, S. Tuning the Photoluminescence of Graphene Quantum Dots through the Charge Transfer Effect of Functional Groups. Acs Nano 2013, 7, 1239-1245.

48. Pan, D. Y.; Zhang, J. C.; Li, Z.; Wu, M. H. Hydrothermal Route for Cutting Graphene Sheets into Blue-Luminescent Graphene Quantum Dots. Adv. Mater. 2010, 22, 734-738.

49. Zhu, S. J.; Zhang, J. H.; Liu, X.; Li, B.; Wang, X. F.; Tang, S. J.; Meng, Q. N.; Li, Y. F.; Shi, C.; Hu, R.; Yang, B. Graphene Quantum Dots with Controllable Surface Oxidation, Tunable Fluorescence and Up-Conversion Emission. Rsc Adv. 2012, 2, 2717-2720.

50. Zong, J.; Zhu, Y. H.; Yang, X. L.; Shen, J. H.; Li, C. Z. Synthesis of Photoluminescent Carbogenic Dots Using Mesoporous Silica Spheres as Nanoreactors. Chem. Commun. 2011, 47, 764-766.

51. Tan, X. Y.; Li, Y. C.; Li, X. H.; Zhou, S. X.; Fan, L. Z.; Yang, S. H. Electrochemical Synthesis of Small-Sized Red Fluorescent Graphene Quantum Dots as a Bioimaging Platform. Chem. Commun. 2015, 51, 2544-2546. 
52. Tang, L. B.; Ji, R. B.; Cao, X. K.; Lin, J. Y.; Jiang, H. X.; Li, X. M.; Teng, K. S.; Luk, C. M.; Zeng, S. J.; Hao, J. H.; Lau, S. P. Deep Ultraviolet Photoluminescence of Water-Soluble Self-Passivated Graphene Quantum Dots. Acs Nano 2012, 6, 5102-5110.

53. Lee, J.; Kim, K.; Park, W. I.; Kim, B. H.; Park, J. H.; Kim, T. H.; Bong, S.; Kim, C. H.; Chae, G.; Jun, M.; Hwang, Y.; Jung, Y. S.; Jeon, S. Uniform Graphene Quantum Dots Patterned from Self-Assembled Silica Nanodots. Nano Lett. 2012, 12, 6078-6083.

54. Ponomarenko, L. A.; Schedin, F.; Katsnelson, M. I.; Yang, R.; Hill, E. W.; Novoselov, K. S.; Geim, A. K. Chaotic Dirac Billiard in Graphene Quantum Dots. Science 2008, 320, 356-358. 55. Song, S. H.; Jang, M.-H.; Chung, J.; Jin, S. H.; Kim, B. H.; Hur, S.-H.; Yoo, S.; Cho, Y.H.; Jeon, S. Highly Efficient Light-Emitting Diode of Graphene Quantum Dots Fabricated from Graphite Intercalation Compounds. Adv. Opt. Mater. 2014, 2, 1016-1023.

56. Ye, R. Q.; Xiang, C. S.; Lin, J.; Peng, Z. W.; Huang, K. W.; Yan, Z.; Cook, N. P.; Samuel, E. L. G.; Hwang, C. C.; Ruan, G. D.; Ceriotti, G.; Raji, A. R. O.; Marti, A. A.; Tour, J. M. Coal as an Abundant Source of Graphene Quantum Dots. Nat. Commun. 2013, 4, 2943. 57. Ciesielski, A.; Samorì, P. Graphene via Sonication Assisted Liquid-Phase Exfoliation. Chem. Soc. Rev. 2014, 43, 381-398.

58. Hernandez, Y.; Nicolosi, V.; Lotya, M.; Blighe, F. M.; Sun, Z. Y.; De, S.; McGovern, I. T.; Holland, B.; Byrne, M.; Gun'ko, Y. K.; Boland, J. J.; Niraj, P.; Duesberg, G.; Krishnamurthy, S.; Goodhue, R.; Hutchison, J.; Scardaci, V.; Ferrari, A. C.; Coleman, J. N. High-yield Production of Graphene by Liquid-Phase Exfoliation of Graphite. Nat. Nanotechnol. 2008, 3, 563-568.

59. Bonaccorso, F.; Lombardo, A.; Hasan, T.; Sun, Z. P.; Colombo, L.; Ferrari, A. C. Production and Processing of Graphene and 2D Crystals. Mater. Today 2012, 15, 564-589. 60. Liang, Y. T.; Hersam, M. C. Highly Concentrated Graphene Solutions via Polymer Enhanced Solvent Exfoliation and Iterative Solvent Exchange. J. Am. Chem. Soc. 2010, 132, 17661-17663.

61. Lotya, M.; Hernandez, Y.; King, P. J.; Smith, R. J.; Nicolosi, V.; Karlsson, L. S.; Blighe, F. M.; De, S.; Wang, Z. M.; McGovern, I. T.; Duesberg, G. S.; Coleman, J. N. Liquid Phase Production of Graphene by Exfoliation of Graphite in Surfactant/Water Solutions. J. Am. Chem. Soc. 2009, 131, 3611-3620.

62. Maragó, O. M.; Bonaccorso, F.; Saija, R.; Privitera, G.; Gucciardi, P. G.; Iati, M. A.; Calogero, G.; Jones, P. H.; Borghese, F.; Denti, P.; Nicolosi, V.; Ferrari, A. C. Brownian Motion of Graphene. Acs Nano 2010, 4, 7515-7523.

63. Torrisi, F.; Hasan, T.; Wu, W. P.; Sun, Z. P.; Lombardo, A.; Kulmala, T. S.; Hsieh, G. W.; Jung, S. J.; Bonaccorso, F.; Paul, P. J.; Chu, D. P.; Ferrari, A. C. Inkjet-Printed Graphene Electronics. Acs Nano 2012, 6, 2992-3006.

64. Kouroupis-Agalou, K.; Liscio, A.; Treossi, E.; Ortolani, L.; Morandi, V.; Pugno, N. M.; Palermo, V. Fragmentation and Exfoliation of 2-Dimensional Materials: a Statistical Approach. Nanoscale 2014, 6, 5926-5933.

65. Durge, R.; Kshirsagar, R. V.; Tambe, P. Effect of Sonication Energy on the Yield of Graphene Nanosheets by Liquid-Phase Exfoliation of Graphite. Procedia Eng. 2014, 97, 14571465.

66. Skaltsas, T.; Ke, X. X.; Bittencourt, C.; Tagmatarchis, N. Ultrasonication Induces Oxygenated Species and Defects onto Exfoliated Graphene. J. Phys. Chem. C 2013, 117, 2327223278. 
67. Coleman, J. N. Liquid Exfoliation of Defect-Free Graphene. Acc. Chem. Res. 2013, 46, 14-22.

68. Bonaccorso, F.; Bartolotta, A.; Coleman, J. N.; Backes, C. 2D-Crystal-Based Functional Inks. Adv. Mater. 2016, 28, 6136-6166.

69. Capasso, A.; Castillo, A. E. D.; Sun, H.; Ansaldo, A.; Pellegrini, V.; Bonaccorso, F. Inkjet Printing of Graphene for Flexible Electronics: An Environmentally-Friendly Approach. Solid State Commun. 2015, 224, 53-63.

70. Yau, H. C.; Bayazit, M. K.; Steinke, J. H. G.; Shaffer, M. S. P. Sonochemical Degradation of N-methylpyrrolidone and its Influence on Single Walled Carbon Nanotube Dispersion. Chem. Commun. 2015, 51, 16621-16624.

71. Niyogi, S.; Hamon, M. A.; Perea, D. E.; Kang, C. B.; Zhao, B.; Pal, S. K.; Wyant, A. E.; Itkis, M. E.; Haddon, R. C. Ultrasonic Dispersions of Single-Walled Carbon Nanotubes. J. Phys. Chem. B 2003, 107, 8799-8804.

72. Riesz, P.; Kondo, T. Free-Radical Formation Induced by Ultrasound and Its Biological Implications. Free Radic. Biol. Med. 1992, 13, 247-270.

73. Katoh, R.; Yokoi, H.; Usuba, S.; Kakudate, Y.; Fujiwara, S. Sonochemical Polymerization of Benzene Derivatives: the Site of the Reaction. Ultrason. Sonochem. 1998, 5, 69-72.

74. Katoh, R.; Tasaka, Y.; Sekreta, E.; Yumura, M.; Ikazaki, F.; Kakudate, Y.; Fujiwara, S. Sonochemical Production of a Carbon Nanotube. Ultrason. Sonochem. 1999, 6, 185-187.

75. Ciesielski, A.; Haar, S.; El Gemayel, M.; Yang, H. F.; Clough, J.; Melinte, G.; Gobbi, M.; Orgiu, E.; Nardi, M. V.; Ligorio, G.; Palermo, V.; Koch, N.; Ersen, O.; Casiraghi, C.; Samorì, P. Harnessing the Liquid-Phase Exfoliation of Graphene Using Aliphatic Compounds: A Supramolecular Approach. Angew. Chem. Int. Ed. 2014, 53, 10355-10361.

76. Haar, S.; Ciesielski, A.; Clough, J.; Yang, H. F.; Mazzaro, R.; Richard, F.; Conti, S.;

Merstorf, N.; Cecchini, M.; Morandi, V.; Casiraghi, C.; Samorì, P. A Supramolecular Strategy to Leverage the Liquid-Phase Exfoliation of Graphene in the Presence of Surfactants: Unraveling the Role of the Length of Fatty Acids. Small 2015.

77. Haar, S.; El Gemayel, M.; Shin, Y. Y.; Melinte, G.; Squillaci, M. A.; Ersen, O.; Casiraghi, C.; Ciesielski, A.; Samorì, P. Enhancing the Liquid-Phase Exfoliation of Graphene in Organic Solvents upon Addition of n-Octylbenzene. Sci.Rep. 2015, 5, 16684.

78. Ferrari, A. C.; Meyer, J. C.; Scardaci, V.; Casiraghi, C.; Lazzeri, M.; Mauri, F.; Piscanec, S.; Jiang, D.; Novoselov, K. S.; Roth, S.; Geim, A. K. Raman Spectrum of Graphene and Graphene Layers. Phys. Rev. Lett. 2006, 97, 187401.

79. Q Qi, B. P.; Hu, H.; Bao, L.; Zhang, Z. L.; Tang, B.; Peng, Y.; Wang, B. S.; Pang, D. W. An Efficient Edge-Functionalization Method to Tune the Photoluminescence of Graphene Quantum Dots. Nanoscale 2015, 7, 5969-5973.

80. Tan, Y.Z.; Yang, B.; Parvez, K.; Narita, A.; Osella, S.; Beljonne, D.; Feng, X.; Müllen, K. Atomically Precise Edge Chlorination of Nanographenes and its Application in Graphene Nanoribbons. Nat. Commun. 2013, 4, 2646-2653.

81. Casiraghi, C. Raman Spectroscopy of Graphene. In Spectroscopic Properties of Inorganic and Organometallic Compounds : Techniques, Materials and Applications, The Royal Society of Chemistry: Cambridge, 2012; Vol. 43, pp 29-56.

82. Backes, C.; Paton, K. R.; Hanlon, D.; Yuan, S.; Katsnelson, M. I.; Houston, J.; Smith, R. J.; McCloskey, D.; Donegan, J. F.; Coleman, J. N. Spectroscopic Metrics Allow in situ 
Measurement of Mean Size and Thickness of Liquid-Exfoliated Few-Layer Graphene Nanosheets. Nanoscale 2016, 8, 4311-4323.

83. Cançado, L. G.; Jorio, A.; Pimenta, M. A. Measuring the Absolute Raman Cross Section of Nanographites as a Function of Laser Energy and Crystallite Size. Phys. Rev. B 2007, 76, 064304.

84. Ribeiro-Soares, J.; Oliveros, M. E.; Garin, C.; David, M. V.; Martins, L. G. P.; Almeida, C. A.; Martins-Ferreira, E. H.; Takai, K.; Enoki, T.; Magalhäes-Paniago, R.; Malachias, A.;

Jorio, A.; Archanjo, B. S.; Achete, C. A.; Cançado, L. G. Structural Analysis of Polycrystalline Graphene Systems by Raman Spectroscopy. Carbon 2015, 95, 646-652.

85. Ferrari, A. C.; Basko, D. M. Raman Spectroscopy as a Versatile Tool for Studying the Properties of Graphene. Nat. Nanotechnol. 2013, 8, 235-246.

86. Ferrari, A. C.; Robertson, J. Interpretation of Raman Spectra of Disordered and Amorphous Carbon. Phys. Rev. B 2000, 61, 14095-14107.

87. Liu, L.; Ryu, S. M.; Tomasik, M. R.; Stolyarova, E.; Jung, N.; Hybertsen, M. S.; Steigerwald, M. L.; Brus, L. E.; Flynn, G. W. Graphene Oxidation: Thickness-Dependent Etching and Strong Chemical Doping. Nano Lett. 2008, 8, 1965-1970.

88. Walter, M. J.; Lupton, J. M. Unraveling the Inhomogeneously Broadened Absorption Spectrum of Conjugated Polymers by Single-Molecule Light-Harvesting Action Spectroscopy. Phys. Rev. Lett. 2009, 103, 167401.

89. Shen, J.; Zhu, Y.; Yang, X.; Li, C. Graphene Quantum Dots: Emergent Nanolights for Bioimaging, Sensors, Catalysis and Photovoltaic Devices. Chem. Commun. 2012, 48, 3686-3699. 90. Zhu, S.; Zhang, J.; Tang, S.; Qiao, C.; Wang, L.; Wang, H.; Liu, X.; Li, B.; Li, Y.; Yu, W.; Wang, X.; Sun, H.; Yang, B. Surface Chemistry Routes to Modulate the Photoluminescence of Graphene Quantum Dots: From Fluorescence Mechanism to Up-Conversion Bioimaging Applications. Adv. Funct. Mater. 2012, 22, 4732-4740.

91. Zhang, W. X.; Dai, D. J.; Chen, X. F.; Guo, X. X.; Fan, J. Y. Red Shift in the Photoluminescence of Colloidal Carbon Quantum Dots Induced by Photon Reabsorption. Appl. Phys. Lett. 2014, 104, 091902.

92. Thiyagarajan, S. K.; Raghupathy, S.; Palanivel, D.; Raji, K.; Ramamurthy, P. Fluorescent Carbon Nano Dots from Lignite: Unveiling the Impeccable Evidence for Quantum Confinement. Phys. Chem. Chem. Phys. 2016, 18, 12065-12073.

93. Chen, S.; Liu, J. W.; Chen, M. L.; Chen, X. W.; Wang, J. H. Unusual Emission Transformation of Graphene Quantum Dots Induced by Self-Assembled Aggregation. Chem. Commun. 2012, 48, 7637-7639.

94. Gierschner, J.; Mack, H. G.; Oelkrug, D.; Waldner, I.; Rau, H. Modeling of the Optical Properties of Cofacial Chromophore Pairs: Stilbenophane. J. Phys. Chem. A 2004, 108, 257-263. 